(C) 1996 IEEE. Personal use of this material is permitted. However, permission to reprint/republish this material for advertising or promotional purposes or for creating new collective works for resale or redistribution to servers or lists, or to reuse any copyrighted component of this work in other works must be obtained from the IEEE.

\title{
A Multisensor Localization Algorithm for Mobile Robots and its Real-Time Experimental Validation
}

\author{
Ph. BONNIFAIT and G. GARCIA \\ Laboratoire d'Automatique de Nantes, URA CNRS 823 \\ Ecole Centrale de Nantes/Université de Nantes, 1 rue de la Noë 44072 Nantes cedex 03, France. \\ Tel: (33) 40-37-16-00; fax : (33) 40-37-25-22; email: Philippe.Bonnifait@lan.ec-nantes.fr.
}

\begin{abstract}
We present a localization system which computes, by extended Kalman filtering, the position and heading of a vehicle using both odometry and the measurement of the relative azimuth angles of known landmarks. Observability of the continuous system is studied and gives information on situations where the performance of the filter may deteriorate. Results of extensive real outdoor experiments with a tyre-type robot moving on a slightly uneven floor are reported. We show that the system achieves a precision of a few centimeters.
\end{abstract}

\section{Introduction}

This paper presents a 2D mobile robot localization system which determines the position and heading of a vehicle by using both odometry and measurements of the azimuth angles of known landmarks. The updating of the odometric estimate using the angular information is performed by and extended Kalman filter (EKF).

A good review of the general framework of sensor integration, to which our work is related, can be found in [11]. The techniques used are similar to those presented in [4], [5] and [7]. But in [4] and [5], quite different sensors are used (a range-finder and a camera respectively). In [7], the sensor only detects landmarks at two fixed azimuth angles, whereas our sensor is a rotating detector and can detect beacons at arbitrary angles.

An important difference between our work and those presented in the fore-mentioned papers is that our system is implemented on an outdoor tyre-type robot which moves on slightly uneven floor (a lawn in our tests). In addition, the field of evolution is fairly large (40x30 square meters) for only three landmarks and can be extended by adding landmarks without any modification of the system.

Moreover, unlike in previous papers, we have also studied the observability of the nonlinear continuous system, independent of the technological aspects of the sensor itself. This has allowed us to determine situations which could generate convergence or precision problems. We have been able to check that, although in some very special cases the performance of the filter can be lower, it never drifts unboundedly. The interest is that these special cases apply to all localization systems which measure azimuth angles, when only three landmarks are visible.

Finally, we report results of real outdoor experiments that show that our system achieves centimeter-level accuracy. The tests involved have been performed by moving along paths, not just checking endpoints in a static situation, as is often done.

\section{The continuous system}

\subsection{System modelling}

Let us consider a three-wheeled, non-holonomic vehicle. In a two-dimensional space, the location of the vehicle can be represented by a triplet $\mathrm{X}=(\mathrm{x}, \mathrm{y}, \theta)$ where $(\mathrm{x}, \mathrm{y})$ is the position of the middle of the wheel base (denoted $\mathrm{M}$ ) and $\theta$ the heading angle with respect to a fixed frame (fig. 1). Let $\mathrm{u}_{1}$ be the translational speed and $\mathrm{u}_{2}$ the rotational speed. The evolution model is classically given by :

$$
\left\{\begin{array}{l}
\dot{\mathrm{x}}=\mathrm{u}_{1} \cdot \cos \theta \\
\dot{\mathrm{y}}=\mathrm{u}_{1} \cdot \sin \theta \text { which can be written as: } \dot{\mathrm{X}}=\mathrm{f}(\mathrm{X}, \mathrm{U}) \\
\dot{\theta}=\mathrm{u}_{2}
\end{array}\right.
$$

with $\mathrm{u}=\left[\begin{array}{ll}\mathrm{u}_{1} & \mathrm{u}_{2}\end{array}\right]^{\mathrm{t}}$ the control vector.

The observations are the azimuth angles $\lambda_{\mathrm{i}}$ of the landmarks $\mathrm{B}_{\mathrm{i}}$, the coordinates of which are denoted $\left(\mathrm{x}_{\mathrm{i}}, \mathrm{y}_{\mathrm{i}}\right)$. The observation equations are (fig. 1) :

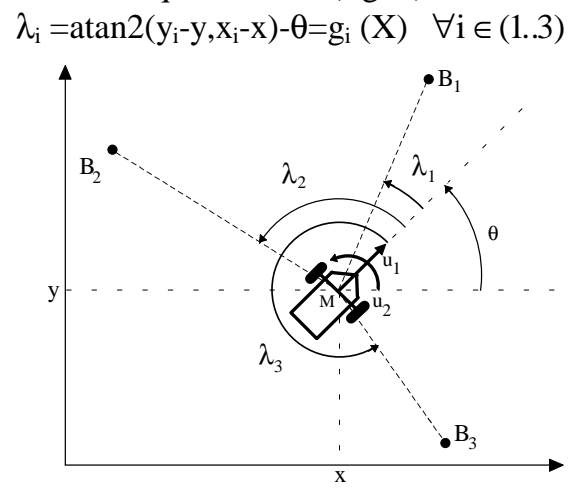

Figure 1 : Azimuth angles definition. 
Equation (2) requires that the reference point of the sensor be set to $\mathrm{M}$.

Three beacons (landmarks) are necessary to compute $\mathrm{X}$, in the reference frame, for a motionless vehicle. The method is described in [1]. We will use three beacons in the sequel. Consider $\lambda=\left[\begin{array}{lll}\lambda_{1} & \lambda_{2} & \lambda_{3}\end{array}\right]^{\mathrm{T}}$.

The observation equation can be rewritten as :

$$
\mathrm{g}(\mathrm{X})=\left[\mathrm{g}_{1}(X) \mathrm{g}_{2}(X) \mathrm{g}_{3}(X)\right]^{\mathrm{t}}
$$

The system formed by the vehicle and the beacons has the following state-space description:

$$
\left\{\begin{array}{l}
\dot{X}=f(X, U) \\
\lambda=g(X)
\end{array}\right.
$$

\subsection{System observability}

In this section, we suppose that the inputs and the outputs of the system are continuously available.

For nonlinear systems, Hermann and Krener [3] related observability to the concept of distinguishability of states with respect to the inputs. They derive a test, known as "rank condition", which implies weak observability. Weak observability intuitively means that the knowledge of the inputs and observations over time is sufficient to determine the initial state of the system, if not among all possible states, at least among all states in a neighborhood of the initial state. In practice, weak observability is usually sufficient. Notice that, contrary to the linear case, nonlinear observability depends on the inputs. let :

$$
\mathbf{O}=\left[\begin{array}{lllllll}
\mathrm{dg}_{1} & \mathrm{dL}_{\mathrm{f}} \mathrm{g}_{1} & \mathrm{dL}_{\mathrm{f}}^{2} \mathrm{~g}_{1} & \ldots & \mathrm{dg}_{3} & \ldots & \mathrm{dL}_{\mathrm{f}}^{2} \mathrm{~g}_{3}
\end{array}\right]
$$

where $d h=\left[\frac{\partial h}{\partial x} \frac{\partial h}{\partial y} \frac{\partial h}{\partial \theta}\right]^{t}$ is the gradient vector, and $\mathrm{L}_{\mathrm{f}} \mathrm{g}=\frac{\partial \mathrm{g}}{\partial \mathrm{x}} \mathrm{f}$ is the Lie derivative of $\mathrm{g}$ with respect to $\mathrm{f}$.

In our case, the Hermann and Krener condition can be expressed as follows : if $\operatorname{rank}(\mathbf{O})=3$, then the system is weakly observable.

First, consider the sub-matrix :

$$
\mathbf{O}_{1}=\left[\begin{array}{lll}
\operatorname{dg}_{1} & \mathrm{dg}_{2} & \mathrm{dg}_{3}
\end{array}\right]
$$

then

$$
\operatorname{dg}_{\mathrm{i}}=\left[\begin{array}{lll}
\frac{\mathrm{y}_{\mathrm{i}}-\mathrm{y}}{\mathrm{D}_{\mathrm{i}}} & \frac{\mathrm{x}-\mathrm{x}_{\mathrm{i}}}{\mathrm{D}_{\mathrm{i}}} & -1
\end{array}\right]^{\mathrm{t}}
$$

with

$$
\mathrm{D}_{\mathrm{i}}=\left(\mathrm{y}-\mathrm{y}_{\mathrm{i}}\right)^{2}+\left(\mathrm{x}-\mathrm{x}_{\mathrm{i}}\right)^{2}
$$

If the determinant of $\mathbf{O}_{1}$ is different from zero then $\operatorname{rank}\left(\mathbf{O}_{1}\right)=3$. The curve $\operatorname{det}\left(\mathbf{O}_{1}\right)=0$ is the circle defined by the three beacons (called (C) on fig. 2).

According to the rank condition, we can conclude that the system is weakly observable anywhere, except, perhaps, on (C). As a matter of fact, since we only considered three columns of $\mathbf{O}_{1}$, we have not proved that the continuous system is not observable on this circle.

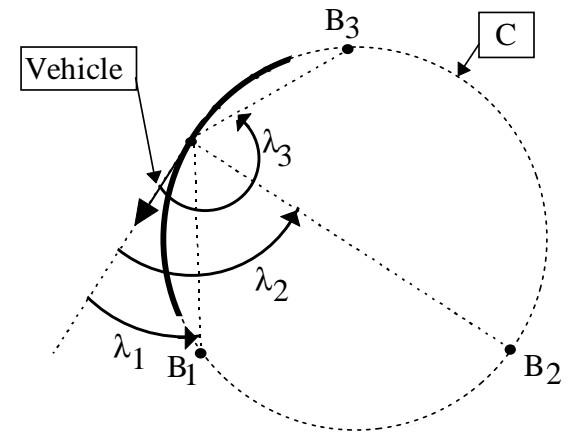

Figure 2 : Problematic trajectory

Consider now the Lie derivative of $g$ with respect to $\mathrm{f}$ :

$$
\mathrm{L}_{\mathrm{f}} \mathrm{g}_{\mathrm{i}}(\mathrm{X})=\mathrm{u}_{1} \cdot\left(\frac{\mathrm{y}_{\mathrm{i}}-\mathrm{y}}{\mathrm{D}_{\mathrm{i}}} \cdot \cos \theta+\frac{\mathrm{x}-\mathrm{x}_{\mathrm{i}}}{\mathrm{D}_{\mathrm{i}}} \cdot \sin \theta\right)-\mathrm{u}_{2}
$$

If $\mathrm{u}_{1}=0$, which means the robot is motionless or rotates around its reference point, then :

$$
\begin{gathered}
\mathrm{L}_{\mathrm{f}} \mathrm{g}_{\mathrm{i}}(\mathrm{X})=-\mathrm{u}_{2} \\
\text { So : } \quad \mathrm{dL}_{\mathrm{f}} \mathrm{g}_{\mathrm{i}}=\mathrm{dL}_{\mathrm{f}}^{2} \mathrm{~g}_{\mathrm{i}}=\overrightarrow{0} \quad \forall \mathrm{i} \in(1 . .3)
\end{gathered}
$$

Thus, in this case, $\operatorname{rank}(\mathbf{O})=\operatorname{rank}\left(\mathbf{O}_{1}\right)$ and therefore, on (C), $\operatorname{rank}(\mathrm{O}) \leq 2$. We can't assert that the system is not observable since the rank condition is only sufficient, nevertheless, by simulations, we have noticed that, in this case, our observer is not able to drive an initial estimation error to zero.

A geometric explanation can be expressed as follows :

On figure 2, we have : $\left\{\begin{array}{l}\lambda_{2}-\lambda_{1}=\alpha_{12} \\ \lambda_{3}-\lambda_{1}=\alpha_{13} \\ \lambda_{3}-\lambda_{2}=\alpha_{23}\end{array}\right.$

where the $\alpha_{\mathrm{ij}}$ are constant parameters depending only on (C). Thus, knowing $\lambda_{1}$ we can compute $\lambda_{2}$ and $\lambda_{3}$. So, on (C), when the mobile does not move, azimuth measurements alone are not sufficient to compute the position and heading.

We could calculate $\mathrm{dL}_{\mathrm{f}} \mathrm{g}_{\mathrm{i}}$ and $\mathrm{dL}_{\mathrm{f}}^{2} \mathrm{~g}_{\mathrm{i}}$ and extract other sub-matrices, but the computations become quickly untractable. Since we do not know whether the system is observable on (C) when $\mathrm{u}_{1}$ and $\mathrm{u}_{2}$ are different from zero, what we will do (see section 5) is test our observer for a mobile running on this possibly difficult trajectory.

\section{Real system state estimation}

\subsection{Algorithm principle}

In fact, the real system differs from the ideal one described in section 2 , in the following ways.

First, the observer will have a discrete-time form, so that estimation will occur at discrete points in time.

Moreover, in section 2, we have supposed that the three azimuth angles were provided simultaneously and continuously (here continuously would be at the sampling rate). 
In fact, the sensor is a CCD detector which rotates with a constant speed. The landmarks (light sources) are detected one at a time and asynchronously, since the angular interval between two landmarks depends on the position and movement of the mobile.

Additionally, we use incremental encoders mounted on the wheels to measure the control vector $u$. Moreover, with these encoders, we can apply the odometric technique which is very helpful for short term location prediction, between the time instants of the goniometric measurements.

The principle of the algorithm presented below relies on such a prediction and uses azimuth angles to update the location of the robot. This multisensor approach takes advantage of sensor redundancy and complementarity. Different variants of this mixed solution (using rangefinders, inertial sensors...) can be found in [4] [5] [6] [7].

The last difference between the real system and the one of section 2 lies in the fact that the projections on the XY plane of the middle of the wheel base (M) and of the rotation axis of the camera (denoted $\mathrm{S}$ ) are different. See figure 3, where the mobile frame $R_{M}$ and the sensor frame $\mathrm{R}_{\mathrm{S}}$ are defined.

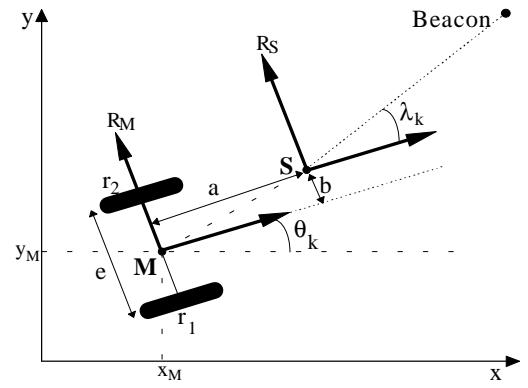

Figure 3 : Notations and translation between $S$ and $M$.

The rotation between $R_{M}$ and $R_{S}$ can be set to null by an appropriate calibration procedure, so that the transform between the two frames reduces to a translation vector $(a, b)$.

If we want to use odometric equations for point $\mathrm{S}$, we need to express the evolution of $S$ as a function of the encoder increments.

\subsection{Evolution model for point $S$}

In this part, we will express the elementary displacements of $S$ as a function of the elementary rotations of the wheels, $\Delta \mathrm{q}_{1 \mathrm{k}}$ and $\Delta \mathrm{q}_{2 \mathrm{k}}$, provided by the optical encoders attached to the right and left wheel.

Let $r_{1}$ and $r_{2}$ denote the radii of the wheels, which can be a bit different from one another for tyre wheels. The wheel base e represents the distance between the middle of the two tyres.

First, we can compute $\Delta \mathrm{D}_{\mathrm{k}}$, the distance travelled by point $\mathrm{M}$, and $\Delta \theta_{\mathrm{K}}$ the angle variation :

$$
\Delta \mathrm{D}_{\mathrm{k}}=\frac{\mathrm{r}_{1} \cdot \Delta \mathrm{q}_{1 \mathrm{k}}+\mathrm{r}_{2} \cdot \Delta \mathrm{q}_{2 \mathrm{k}}}{2} \quad \Delta \theta_{\mathrm{k}}=\frac{\mathrm{r}_{1} \cdot \Delta \mathrm{q}_{1 \mathrm{k}}-\mathrm{r}_{2} \cdot \Delta \mathrm{q}_{2 \mathrm{k}}}{\mathrm{e}}
$$

We will use the following notations : $X_{M}$ and $X_{S}$ correspond to time index $\mathrm{k}$ and, $\mathrm{X}_{\mathrm{M}+1}$ and $\mathrm{X}_{\mathrm{S}+1}$ to time index $k+1$. Note that $\theta_{S}=\theta_{M}=\theta_{k}$.

Suppose we know $X_{M}$, we can calculate $X_{M+1}$ by approximating the trajectory as a sequence of constant curvature segments of length $\Delta \mathrm{D}_{\mathrm{k}}$. Moreover, if $\Delta \theta_{\mathrm{k}}$ is small, then [8] :

$$
\left\{\begin{array}{l}
\mathrm{x}_{\mathrm{M}+1} \approx \mathrm{x}_{\mathrm{M}}+\Delta \mathrm{D}_{\mathrm{k}} \cdot \cos \left(\theta_{\mathrm{k}}+\Delta \theta_{\mathrm{k}} / 2\right) \\
\mathrm{y}_{\mathrm{M}+1} \approx \mathrm{y}_{\mathrm{M}}+\Delta \mathrm{D}_{\mathrm{k}} \cdot \sin \left(\theta_{\mathrm{k}}+\Delta \theta_{\mathrm{k}} / 2\right) \\
\theta_{\mathrm{k}+1}=\theta_{\mathrm{k}}+\Delta \theta_{\mathrm{k}}
\end{array}\right.
$$

Considering figure 3 , we have :

$$
\begin{aligned}
& \left\{\begin{array}{l}
\mathrm{x}_{\mathrm{S}}=\mathrm{x}_{\mathrm{M}}+\mathrm{a} \cdot \cos \theta_{\mathrm{k}}-\mathrm{b} \cdot \sin \theta_{\mathrm{k}} \\
\mathrm{y}_{\mathrm{S}}=\mathrm{y}_{\mathrm{M}}+\mathrm{a} \cdot \sin \theta_{\mathrm{k}}+\mathrm{b} \cdot \cos \theta_{\mathrm{k}}
\end{array}\right. \\
\mathrm{x}_{\mathrm{S}+1}= & \mathrm{x}_{\mathrm{M}+1}+\mathrm{a} \cdot \cos \theta_{\mathrm{k}+1}-\mathrm{b} \cdot \sin \theta_{\mathrm{k}+1} \\
\approx & \mathrm{x}_{\mathrm{M}}+\Delta \mathrm{D} \cdot \cos \left(\theta_{\mathrm{k}}+\Delta \theta_{\mathrm{k}} / 2\right) \\
& +\mathrm{a} \cdot \cos \left(\theta_{\mathrm{k}}+\Delta \theta_{\mathrm{k}}\right)-\mathrm{b} \cdot \sin \left(\theta_{\mathrm{k}}+\Delta \theta_{\mathrm{k}}\right)
\end{aligned}
$$

We want to express $\mathrm{x}_{\mathrm{S}+1}$ as a function of $\mathrm{x}_{\mathrm{S}}$. This we achieve by a first order Taylor expansion :

$$
\begin{aligned}
\mathrm{x}_{\mathrm{S}+1} \approx & \mathrm{x}_{\mathrm{S}}+\Delta \mathrm{D} \cdot \cos \left(\theta_{\mathrm{k}}+\Delta \theta_{\mathrm{k}} / 2\right) \\
& -\Delta \theta_{\mathrm{k}} \cdot\left(\mathrm{a} \cdot \sin \theta_{\mathrm{k}}+\mathrm{b} \cdot \cos \theta_{\mathrm{k}}\right)
\end{aligned}
$$

Repeating the same operation with $\mathrm{y}_{\mathrm{S}}$, we then find :

$$
\left\{\begin{aligned}
\mathrm{x}_{\mathrm{S}+1} \approx & \mathrm{x}_{\mathrm{S}}+\Delta \mathrm{D} \cdot \cos \left(\theta_{\mathrm{k}}+\Delta \theta_{\mathrm{k}} / 2\right) \\
& -\Delta \theta_{\mathrm{k}} \cdot\left(\mathrm{a} \cdot \sin \theta_{\mathrm{k}}+\mathrm{b} \cdot \cos \theta_{\mathrm{k}}\right) \\
\mathrm{y}_{\mathrm{S}+1} \approx & \mathrm{y}_{\mathrm{S}}+\Delta \mathrm{D} \cdot \sin \left(\theta_{\mathrm{k}}+\Delta \theta_{\mathrm{k}} / 2\right) \\
& +\Delta \theta_{\mathrm{k}} \cdot\left(\mathrm{a} \cdot \cos \theta_{\mathrm{k}}-\mathrm{b} \cdot \sin \theta_{\mathrm{k}}\right. \\
\theta_{\mathrm{k}+1}= & \theta_{\mathrm{k}}+\Delta \theta_{\mathrm{k}}
\end{aligned}\right.
$$

This system can be rewritten as : $\mathrm{X}_{\mathrm{S}+1}=\mathrm{F}\left(\mathrm{X}_{\mathrm{S}}, \Delta \mathrm{qk}_{\mathrm{k}}\right)$

where $\Delta \mathrm{q}_{\mathrm{k}}=\left[\begin{array}{ll}\Delta \mathrm{q}_{1 \mathrm{k}} & \Delta \mathrm{q}_{2 \mathrm{k}}\end{array}\right]^{\mathrm{t}}$ and $\mathrm{F}$ is a vector-valued function $: \mathfrak{R}^{3} \times \mathfrak{R}^{2} \rightarrow \mathfrak{R}^{3}$

The model obtained this way is a good approximation of reality when $\Delta \theta_{\mathrm{k}}$ is small, which requires that the sampling period be sufficiently small with respect to the vehicle translational and rotational speed. The simplicity of this model makes the calculations of the Jacobian matrices of the EKF tractable.

\subsection{Discrete Kalman filtering formulation}

Let us consider now the nonlinear stochastic system with state-space description :

$$
\left\{\begin{aligned}
\mathrm{X}_{\mathrm{S}+1} & =\mathrm{F}\left(\mathrm{X}_{\mathrm{S}}, \Delta \mathrm{q}_{\mathrm{k}}\right)+\alpha_{\mathrm{k}} \\
\lambda_{\mathrm{k}} & =\mathrm{g}_{\mathrm{i}}\left(\mathrm{X}_{\mathrm{S}}\right)+\beta_{\mathrm{k}} \text { with } \mathrm{i}=1,2 \text {, or } 3
\end{aligned}\right.
$$

One can note that the observation equation is now scalar but non stationary.

$\alpha_{\mathrm{k}}$ and $\beta_{\mathrm{k}}$ are, respectively, system and observation noises. We suppose they are independent sequences of zero-mean Gaussian white noise such that $\operatorname{Var}\left(\alpha_{\mathrm{k}}\right)=\mathrm{Q}_{\alpha}$ and $\operatorname{Var}\left(\beta_{\mathrm{k}}\right)=\sigma_{\lambda}^{2}$.

$\operatorname{Var}\left(\alpha_{k}\right)$ represents the effects of slippage or dragging on the ground, plus the effects of errors on robot parameters such as $r_{1}, r_{2}$ and e. 
Step 1: Between two azimuth readings, a "high" frequency $(20 \mathrm{~Hz}$ as compared to the frequency of the azimuth readings $\approx 0.5 \mathrm{hz}$ ) state and error prediction phase occurs. For updating $\mathrm{X}_{\mathrm{S}}$, we use the previous estimate (denoted $\hat{X}_{\mathrm{S} / \mathrm{S}}$ ) and the new measurement $\Delta \mathrm{q}_{\mathrm{k}}$ :

$$
\hat{\mathrm{X}}_{\mathrm{S}+1 / \mathrm{S}}=\mathrm{F}\left(\hat{\mathrm{X}}_{\mathrm{S} / \mathrm{S}}, \Delta \mathrm{q}_{\mathrm{k}}\right)
$$

If we suppose that the two encoders are identical and independent, the covariance matrix of $\Delta \mathrm{q}_{\mathrm{k}}$ can be written as : $\operatorname{Var}\left(\Delta q_{k}\right)=\sigma^{2} I_{22}$, where $I_{22}$ is the identity matrix.

The covariance matrix $\mathrm{P}_{\mathrm{k}+1 / \mathrm{k}}$ describes the uncertainty related to $\hat{\mathrm{X}}_{\mathrm{S}+1 / \mathrm{S}}$. Its expression is obtained using the first order Taylor expansion of $\mathrm{F}$, under the reasonable assumption that random vectors $X_{S}$ and $\Delta q_{k}$ are independent. Thus, the three vectors $X_{S}, \Delta q_{k}$ and $\alpha_{k}$ are not correlated and we can apply the result given in [9] :

$$
\mathrm{P}_{\mathrm{k}+1 / \mathrm{k}} \approx \mathrm{A}_{\mathrm{k}} \cdot \mathrm{P}_{\mathrm{k} / \mathrm{k}} \cdot \mathrm{A}_{\mathrm{k}}^{\mathrm{t}}+\sigma^{2} \cdot \mathrm{B}_{\mathrm{k}} \cdot \mathrm{B}_{\mathrm{k}}^{\mathrm{t}}+\mathrm{Q}_{\alpha}
$$

Where the Jacobian $A_{k}=\left[\frac{\partial F}{\partial X}\right]$ and $B_{k}=\left[\frac{\partial F}{\partial \Delta q}\right]$ are evaluated respectively at $\hat{\mathrm{X}}_{\mathrm{S} / \mathrm{S}}$ and $\Delta \mathrm{q}_{\mathrm{k}}$.

Step 2: When an azimuth measurement occurs (approximately every $2 \mathrm{~s}$ ), odometry is updated : it is the estimation phase

We first compute the Jacobian $C_{k}=\left[\frac{\partial g_{i}}{\partial X}\right]$ at $\hat{X}_{S+1 / S}$.

Then, we obtain the Kalman gain vector :

$$
\mathrm{K}=\mathrm{P}_{\mathrm{k}+1 / \mathrm{k}} \cdot \mathrm{C}_{\mathrm{k}}^{\mathrm{t}} \cdot\left(\mathrm{C}_{\mathrm{k}} \cdot \mathrm{P}_{\mathrm{k}+1 / \mathrm{k}} \cdot \mathrm{C}_{\mathrm{k}}^{\mathrm{t}}+\sigma_{\lambda}^{2}\right)^{-1}
$$

The term to invert being a scalar, the inversion is not a problem. The updated estimate and its associated covariance matrix are then classically given by :

$$
\begin{gathered}
\hat{\mathrm{X}}_{\mathrm{S}+1 / \mathrm{S}+1}=\hat{\mathrm{X}}_{\mathrm{S}+1 / \mathrm{S}}+\mathrm{K} \cdot\left(\lambda_{\mathrm{k}}-\mathrm{g}_{\mathrm{i}}\left(\hat{\mathrm{X}}_{\mathrm{S}+1 / \mathrm{S}}\right)\right) \\
\mathrm{P}_{\mathrm{k}+1 / \mathrm{k}+1}=\left(\mathrm{I}-\mathrm{K} \cdot \mathrm{C}_{\mathrm{k}}\right) \cdot \mathrm{P}_{\mathrm{k}+1 / \mathrm{k}}
\end{gathered}
$$

Equation (23) shows that $\hat{\mathrm{X}}_{\mathrm{S}+1 / \mathrm{S}+1}$ is corrected proportionally to the difference between the measured azimuth angle $\lambda_{\mathrm{k}}$ and the expected angle $\mathrm{g}_{\mathrm{i}}\left(\hat{\mathrm{X}}_{\mathrm{S}+1 / \mathrm{S}}\right)$.

The radii of the wheels $\left(r_{1}\right.$ and $\left.r_{2}\right)$ and the wheel base (e) are crucial parameters of the robot model. In practice, the radii of the wheels are not easy to determine, especially when rubber tyres are used. Furthermore, they may even be subject to slow evolutions over time, due to temperature, terrain modifications, tyre pressure, etc.

A solution to this type of problems is to identify these parameters on-line, starting from an initial estimate. This can be accomplished in the context of Kalman filtering by adding the parameters to be identified to the state vector [10]. The new state vector is then :

$$
X=\left[\begin{array}{lllll}
x_{S} & y_{S} & \theta_{k} & r_{1_{k}} & r_{2_{k}}
\end{array}\right]^{t}
$$

The evolution model for $r_{1}$ and $r_{2}$ is a constant. This does not mean that states will not change. Evolution will be made possible by adding a random noise (denoted $\alpha_{\mathrm{ri}}$ ) to this model :

$$
\left\{\begin{array}{l}
r_{1_{k+1}}=r_{1_{k}}+\alpha_{r_{1}} \\
r_{2_{k+1}}=r_{2 k}+\alpha_{r_{2}}
\end{array}\right.
$$

Simulations [2] prove that the filter is able to compensate for an initial error on the radii and even to track slow variations. In [2], we have investigated the robustness of the filter, i.e. its reaction to a calibration error on a parameter which is not part of the state vector, namely the wheel base. In this case, the radii converge, but not towards the real values, yielding a (local) "equivalent robot model".

Furthermore, we have shown the influence of radius identification on the overall quality of the localization process, comparing the behaviour of our filter with one that does not perform radius identification. The output location variables of the filter we obtain, contrary to the filter that does not identify the radii, are unbiased and the amplitudes of the oscillations are greatly reduced, in the case of error-corrupted parameters.

When the sensor detects a landmark, the filter needs to associate the angle with an observation equation, in other words, the system needs to know which beacon has been read. The association technique we choose relies on the dimensionsless Mahalanobis distance, denoted D [11].

$$
\mathrm{D}=(\lambda-\hat{\lambda})^{2} \cdot\left(\mathrm{C}_{\mathrm{k}} \cdot \mathrm{P}_{\mathrm{k}+1 / \mathrm{k}} \cdot \mathrm{C}_{\mathrm{k}}^{\mathrm{t}}+\sigma_{\lambda}^{2}\right)^{-1}
$$

Note that the last term has been previously computed in (22). If $\mathrm{D}$ is smaller than a predefined threshold $\mathrm{D}_{0}$ then the prediction and the observation are consistent, otherwise the measure is rejected. In our case $D_{0}=5$, which corresponds to 2.24 standard deviations.

\section{Real outdoor experiments}

Performed with a 486 PC, real experiments have been run on an outdoor test-track marked out with three beacons (see fig. 4). Fifteen reference marks have been located by surveyors, in a local frame, so that white strings can be stretched on the grass to materialize straight lines of known equations.

The principle of our experiments is to make the robot track those reference paths and to compare the EKF estimation results with reality. A CCD linear camera, at the front of the robot, extracts the deviation between it's optical axis and the white line to be tracked (see fig. 4).

An identification procedure, not described here, gave us the following results for the geometrical parameters of figure $3: a \approx-314.5 \pm 2.5 \mathrm{~mm}$ and $b \approx 0 \pm 2.5 \mathrm{~mm}$. So, after a short initial phase, we can admit that point $S$ is above the white line, as shown on figure 4. 

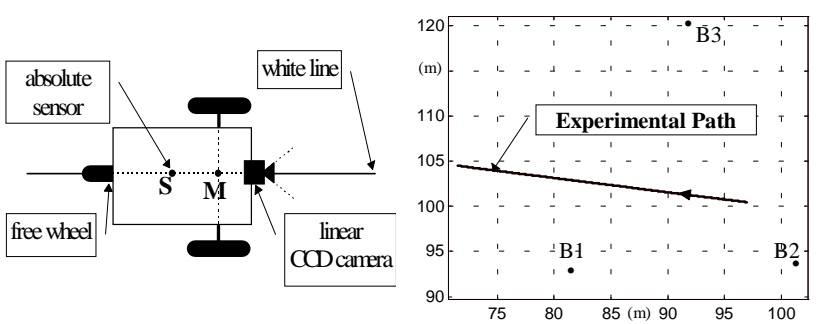

Figure 4 : Experimental setup, beacons locations and path

In the following test, the robot moves along a straight line, which starts in the triangle formed by the beacons and goes comparatively far outside (fig. 4).

Before the robot moves, we compute $\hat{\mathrm{X}}_{\mathrm{S}_{0}}$ using a triangulation method [1] and angles from the CCD sensor. The initial covariance $\mathrm{P}_{0}$ is a diagonal matrix, the diagonal of which is $\left[\begin{array}{lllll}1 & 1 & 0.5 & 10^{-4} & 10^{-4}\end{array}\right]$ SI units.

The variances of the sensors are $\sigma_{\lambda}^{2}=2.8 .10^{-6} \mathrm{rad}^{2}$ and $\sigma^{2}=10^{-9} \operatorname{rad}^{2}$ We have performed extensive postprocessing with real data to tune up noise variances : $Q_{\alpha}$ is a diagonal matrix, the diagonal of which is $\left[\begin{array}{lllll}0 & 0 & 0 & 10^{-9} & 10^{-9}\end{array}\right]$ SI units.

One can remark that the model noise on $(\mathrm{x}, \mathrm{y}, \theta)$ is equal to zero. We have noticed that a non-zero noise on $(\mathrm{x}, \mathrm{y}, \theta)$ deteriorates the radii identification process. Moreover, setting these noises to zero is not a problem since the term $\sigma^{2} \cdot B_{k} \cdot B_{k}^{t}$ has a similar effect as $Q_{\alpha}$ on the position and heading (see equation (21)). In addition, the error ellipsoid generated has a variable orientation, depending on the inputs to the system. This is much more consistent with reality than the constant-orientation ellipsoid generated by $\mathrm{Q}_{\alpha}$.

As we know the equation of the real path, we can compute the lateral error as the distance from the estimated position to the real path (fig. 5). The heading error is the difference between the estimated heading and the real (here constant) heading (fig. 6). On both figures, the signal labeled "with EKF" (resp. "without EKF") denotes the error on the filter output (resp. the result of odometry without using any azimuth measurement).

As was predictable, the results obtained without using angular measurements tend to drift. At the end of the trajectory, the error is roughly one meter. On the contrary, the output error of the filter is bounded (less than $3 \mathrm{~cm}$ lateral error and less than $0.5^{\circ}$ heading error).

At the end of the trajectory, results start to deteriorate. This accuracy deterioration is due to the fact that beacons are all behind the vehicle and, hence, in a bad configuration.

These results prove that the EKF has a very good behaviour. One should note that the accuracy of the reference marks and beacons positioning is about one centimeter. Moreover, it's difficult to guarantee that the string is perfectly stretched out and the robot, when following the line, oscillates a little $(<1 \mathrm{~cm})$. Finally, because the sensor is about $1.9 \mathrm{~m}$ above the ground, one degree of roll angle generates a three-centimeter lateral error (our test-track is a lawn and not perfectly planar).

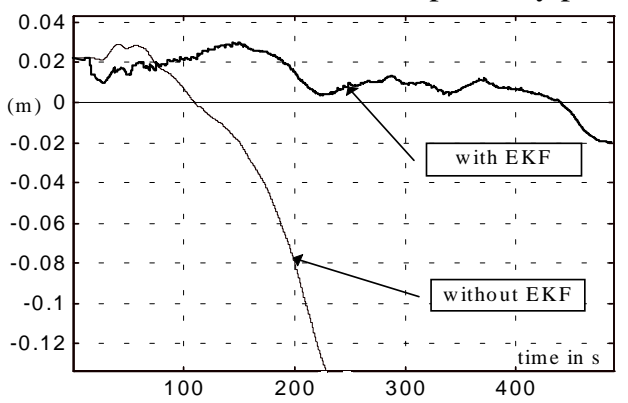

Figure 5 : lateral errors $($ speed $\approx 0.2 \mathrm{~m} / \mathrm{s}$ )

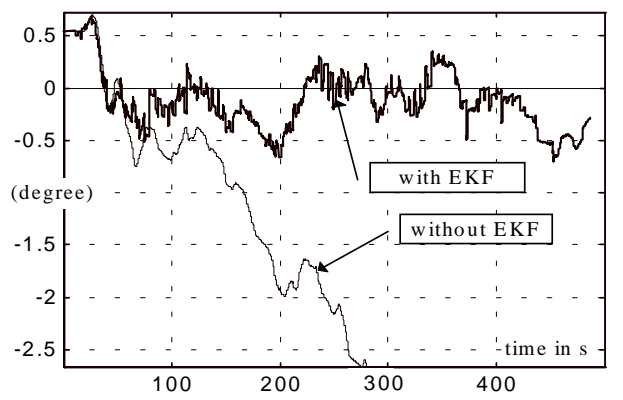

Figure 6 : heading errors (speed $\approx 0.2 \mathrm{~m} / \mathrm{s}$ )

On figure 7 , we show the repeatability of the lateral error for three tests performed along the same path.

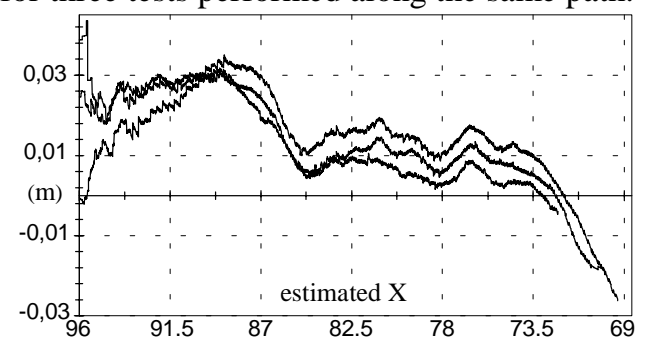

Figure 7 : repeatability of the lateral error $($ speed $\approx 0.2 \mathrm{~m} / \mathrm{s}$ )

Considering these results, it is clear that the lateral error is not a random signal due to measurement noise only. Most probably, it is largely due to the terrain generating the same roll angle at each test. Except for the short transient phase which corresponds to the effect of discrepancies between initial errors, the three curves fit in a narrow one-centimeter wide region. One centimeter is probably closer to the standard deviation of the real lateral error, but this requires testing with a more appropriate setup.

\section{Study of the filter convergence on (C)}

In section 2 , the observability of the system has not been proved on $(\mathrm{C})$ for non-zero inputs.

In practice, such a trajectory is unlikely to occur. In a typical application the robot moves in the triangle formed by the three beacons. When out of this triangle, it typically 
uses a new set of closer beacons. Nevertheless, we will study the behaviour of our filter on an arc of (C) (see fig. 2 where the tested trajectory is in bold).

In the following simulation, we gave an erroneous initial estimate to the filter with position and heading errors. Lateral error is the smallest distance between the estimate and (C). This allows to check whether the estimate remains on the reference path or not. We also calculate the distance between the estimated and real positions.

Through these experiments (fig. 8), one can see that the filter converges quickly : after a small number of rotations, errors have zero means. But the amplitude of the error is larger than on any other path. Nevertheless, errors remain bounded.

The convergence of the filter is due to the evolution model, namely odometry.

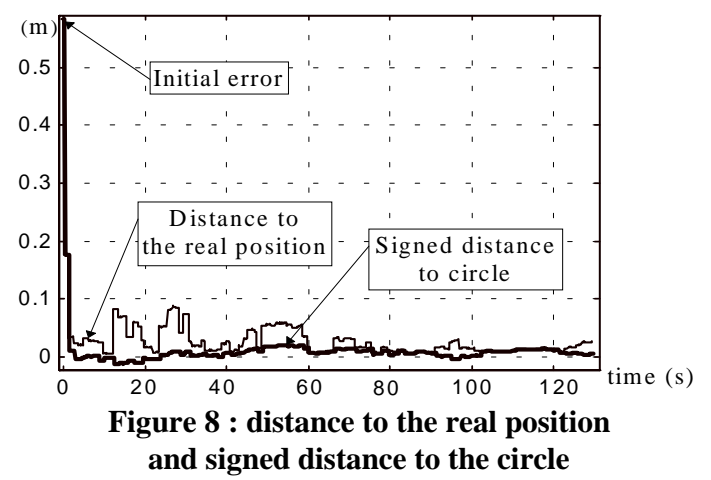

\section{Conclusion}

In this paper, we have dealt with several aspects of a 2D (position and heading) localization system.

We have applied nonlinear theory results to predict whether an observer could converge. This a priori study is very helpful when we can conclude the system is not observable : the system needs more (or more appropriate) sensors and it is useless to try to find a solution.

Nevertheless, this step cannot guarantee that there exists an observer which computes the state when the state is claimed to be observable.

Another interest of such a theoretical study is to determine situations where any localizer based on the same continuous system will undergo difficulties, whatever the technological and algorithmical solutions.

The practical part of the work involves simulations, post-processing of real data and real-time localization on an outdoor tyre-type vehicle moving on uneven floor.

This last aspect is of particular interest since it generates model errors and other disturbances. Among others, errors due to the terrain are evidenced by repeatability tests. The conclusion is that our system behaves particularly well and is robust to these types of disturbances.
Finally, the precision we get, although difficult to measure precisely with the current experimental setup, is clearly centimeter-level. Considering the conditions, this is a very encouraging result.

The next work planned is now to test the precision of the system in more appropriate conditions. This should involve testing our system on the special test-bed SESSYL designed by the LCPC (Laboratoire Central des Ponts et Chaussées, the French institution in charge of applied research in road construction), in the framework of an existing cooperation between the LCPC and our laboratory.

\section{References}

[1] J.-F. Le Corre and G. Garcia, "Real-time determination of the location and speed of mobile robots running on non-planar surfaces". IEEE Int. Conf. on Robotics and Automation. Nice, May 1992.

[2] G. Garcia, Ph. Bonnifait and J.-F Le Corre, "A multisensor fusion localization algorithm with selfcalibration of error-corrupted mobile robot parameters", Int. Conf. on Advanced Robotics, Barcelona, Sept. 1995.

[3] R. Hermann, A.J. Krener, "Nonlinear controllability and observability", IEEE Transactions On Automatic Control, vol. AC-22, $\mathrm{n}^{\circ}$ 5, Oct. 1977.

[4] C. Durieu, J. Opderbecke and G. Allègre, "A data fusion application for location of a mobile robot using an odometer and a panoramic laser telemeter", Intelligent Autonomous Systems 3, Pittsburgh, Feb 1993.

[5] F. Chenavier and J.L. Crowley, "Position estimation for a mobile robot using vision and odometry", IEEE Int. Conf. on Robotics and Automation. Nice, May 1992.

[6] J. Vaganay, M.J. Aldon and A. Fournier, "Mobile robot attitude estimation by fusion of inertial data", IEEE Int. Conf. on Robotics and Automation, Vol.1, Atlanta, May 1993.

[7] T. Nishizawa, A. Ohya and S. Yuta, "An implementation of on-board position estimation for mobile robot", IEEE Int. Conf. on Robotics and Automation, Nagoya, May 1995.

[8] C. Ming Wang, "Location estimation and uncertainty analysis for mobile robots", IEEE Int. Conf. on Robotics and Automation, Philadelphia, April 1988.

[9] R. Smith, M. Self and P. Cheeseman, "Estimating Uncertain Spatial Relationships in Robotics", 1988 Elsevier Science Publishers, Uncertainty in Artificial Intelligence, Vol 2.

[10] C.K. Chui, G.Chen, "Kalman filtering with realtime applications", second edition, Springer-Verlag, 1991.

[11] H.F. Durrant-Whyte, "Sensor models and multisensor integration", Journal of Robotics Research, Vol. 7, N6 Dec. 1988. 